\title{
Sidebranching induced by external noise in solutal dendritic growth
}

\author{
R. González-Cinca* and L. Ramírez-Piscina \\ Departament de Física Aplicada, Universitat Politècnica de Catalunya, Campus Nord UPC, Mòdul B5, E-08034 Barcelona, Spain \\ J. Casademunt and A. Hernández-Machado \\ Departament ECM, Facultat de Física, Universitat de Barcelona, Diagonal 647, E-08028 Barcelona, Spain
}

(Received 22 September 2000; published 16 April 2001)

\begin{abstract}
We have studied sidebranching induced by fluctuations in dendritic growth. The amplitude of sidebranching induced by internal (equilibrium) concentration fluctuations in the case of solidification with solutal diffusion is computed. This amplitude turns out to be significantly smaller than values reported in previous experiments. The effects of other possible sources of fluctuations (of an external origin) are examined by introducing nonconserved noise in a phase-field model. This reproduces the characteristics of sidebranching found in experiments. Results also show that sidebranching induced by external noise is qualitatively similar to that of internal noise, and it is only distinguished by its amplitude.
\end{abstract}

DOI: 10.1103/PhysRevE.63.051602

PACS number(s): 68.70.+w, 81.10.Aj, 64.70.Dv, 05.40.-a

\section{INTRODUCTION}

Dendritic growth in nonequilibrium systems has been extensively studied during the last few years [1-7]. A feature which remains a main point of interest is the study of sidebranching, which is the secondary branches that appear at both sides of the main dendrite. The question of how its frequency and amplitude are determined has not yet been fully solved. Two scenarios have been proposed to explain the origin of sidebranching. One of them states that periodic deterministic oscillations at the tip [8-10] generate correlated branches on both flanks of the dendrite $[8,9]$. A possible source of these tip oscillations was suggested in Ref. [11], where it was argued that the spreading rate of the wave packet that characterizes sidebranching might become large enough so that the tip could undergo oscillations or other instabilities. This is predicted to occur in the limit of small surface tension anisotropy. The other scenario proposes that sidebranching is due to selective amplification of fluctuations near the tip of the dendrite [12-27]. In this case, branches appear to be mostly uncorrelated. In this paper we will study this second scenario by means of a phase-field model [25$40]$ and, in particular, we will focus on the issue of an external vs internal origin of the noise.

In a frame of reference moving with the tip of the dendrite, sidebranching can be seen as a wave that propagates along the dendrite away from the tip at the same velocity as the tip. An appropriate characterization is provided by the amplitude and wavelength. Barber et al. [14] studied the evolution of time-dependent deformations of the needle crystal (Ivantsov) solution of the two-dimensional symmetric model of solidification in the limit of small Pèclet number within a WKB approximation. The amplitude of a localized wave packet grows exponentially as $z^{1 / 4}$, where $z$ is measured from the tip along the symmetry axis of the dendrite, as the packet moves down, provided the initial packet con-

\footnotetext{
*Author to whom correspondence should be addressed. Email address: ricard@fa.upc.es
}

tains modes of arbitrarily small frequencies. Moreover, the wavelength of the packet increases as $z^{1 / 4}$. Pieters [15] obtained the same amplitude and wavelength dependence on $z$ as in Ref. [14] both analytically and by numerical integration of the boundary-layer model. Langer [16] concluded, from a similar analysis to that of Ref. [14] but performed in three dimensions, that noise of some kind can be the origin of sidebranching, but that thermal fluctuations are not strong enough to entirely explain the phenomena. However, more recently, Brener and Temkin [17] considered anisotropic needle crystals in three dimensions and concluded that experimentally observed sidebranching could be explained by considering noise of a thermal origin. The growth of the sidebranching amplitude was found to behave exponentially as a function of $z^{2 / 5}$, which is faster than the $z^{1 / 4}$ dependence obtained in the axisymmetric case [16]. The sidebranching wavelength was found to be a function of $z^{1 / 5}$, very similar to that obtained in the axisymmetric case. Dougherty et al. [18] studied sidebranching in $\mathrm{NH}_{4} \mathrm{Br}$ dendrites, where rather uncorrelated variations in phase and amplitude of the branches were observed. They determined the amplitude of the sidebranching and its mean wavelength by looking at the power spectrum of the data obtained by measuring the half-width of the dendrite at a fixed distance $z$ from the tip at different times. The behavior of the amplitude was qualitatively similar to that predicted in Ref. [14] up to a certain value of $z$, after which the linear theory is presumably no longer valid. An equivalent exponential growth of the amplitude with $s^{1 / 4}$, where $s$ is the arclength, was also found in Ref. [19]. However, in Ref. [18] no variation of the mean frequency in the spectral peak was obtained for different $z$. Finally, Dougherty et al. also observed that side branches separated by more than about six times the mean wavelength were uncorrelated. Weak correlation between opposite sides of the dendrite when no external forcing was applied to it was also found in Ref. [20]. The common feature of all these experiments is that sidebranching appears to be due to the selective amplification of natural noise and not to the existence of some intrinsic oscillation or limit cycle.

Bisang and Bilgram $[21,22]$ found quantitative agreement 
between the predictions for the linear regime in Ref. [17] and their results in experiments on xenon dendrites in three dimensions. They concluded that Brener and Temkin's theory correctly describes the sidebranching behavior of dendrites for any pure substance with cubic symmetry and thus thermal noise was concluded to be the origin of the sidebranching observed in their experiments.

In the last decade there has been an increasing use of phase-field models to deal with dendritic growth problems. They are a very useful and practical tool to simulate such kind of processes and a good alternative to the integrodifferential equation which can be derived from the classical sharp-interface model. In the phase-field models an order parameter or phase field $\phi$ is defined, which avoids tracking the interface and naturally includes the physical boundary conditions at the interface.

Up to now, few studies of sidebranching phenomena with the phase-field model have been carried out. It has been shown that the inclusion of a noise source in the phase-field model equations enhances the emergence of uncorrelated sidebranching [28] without affecting the velocity and radius of the tip [29]. Moreover, when the dendrite tip is periodically forced, the sidebranching appears to be correlated at both sides of the dendrite [33] as has been observed in some experiments $[20,24]$. In particular, sidebranching can be regulated by spatially homogeneous time-periodic variation of the melting point induced by oscillations in the external pressure or by periodic heating generated by a dissipative electric current [40].

The deepest insight into the study of sidebranching with a phase-field model have been carried out recently in Ref. [26]. They included thermal noise in a two-dimensional phasefield model of solidification controlled by heat diffusion in a way which was consistent with both bulk and interfacial equilibrium fluctuations, as has been done previously with the sharp-interface model equations $[41,42]$. Karma and Rappel [26] obtained good quantitative agreement between the computed sidebranching amplitude as a function of distance to the tip and the prediction of the linear WKB theory for anisotropic crystals in two dimensions. Sidebranching wavelength very close to the tip was found to increase with $z$ faster than predicted by the WKB theory, but this could be explained after considering that perturbations generally get stretched as they travel along the sides of curved fronts. Further from the tip, the value of the sidebranching wavelength saturates.

Although there is general agreement in that thermal (internal) fluctuations are enough to explain the amplitude of the dendritic sidebranching, one should be aware that evidence along these lines has been achieved in experiments of heat-controlled solidification of pure substances. As up to now there is a lack of predictions of sidebranching amplitudes for solutal dendrites, experiments of these dendrites can only show qualitative agreement with theoretical results. In this paper we address the question of sidebranching characteristics in the presence of external vs internal fluctuations. First of all, we obtain a prediction of the effects of internal noise on sidebranching amplitudes for solutal dendrites. A comparison of the theory with available quantitative experi- mental results [18] shows that there are serious indications that in some experiments internal thermodynamical fluctuations could not account for observed sidebranching activity. In this case some other source of fluctuations, of an external origin, should be called on. Some of the consequences derived from adding a nonconserved noise source in a twodimensional phase-field model are examined. This noise is of a different nature to what one should employ to provide an account of internal fluctuations [25-27,41,42]. However, our simulations qualitatively reproduce the properties of the noise-induced sidebranching derived analytically $[17,26]$ and observed experimentally $[18,19]$. We conclude that although thermal noise is not always the main origin of dendritic sidebranching, its qualitative characteristics are common for noise-induced sidebranching independently of its origin. A detailed quantitative study of sidebranching activity could therefore be useful to elucidate the origin of the noise in specific experiments.

In Sec. II we predict the sidebranching amplitudes for solutal dendrites with thermal fluctuations. In Sec. III the model and the numerical method used to solve the equations are described. Numerical results on the behavior of the sidebranching characteristics as well as comparison with theoretical predictions and experimental results are described in Sec. IV. Conclusions derived from these results are outlined in Sec. V.

\section{SIDEBRANCHING AMPLITUDE IN SOLUTAL SOLIDIFICATION}

Available theoretical predictions on sidebranching amplitudes have been formulated for dendrites grown from a pure substance and controlled by heat diffusion. Here we will consider dendrites appearing in isothermal growth of mixtures controlled by diffusion of the solute. We start from the Langevin formalism for solidification due to Karma [41,42]. In this formalism the usual sharp-interface model for solidification is completed with noise terms constructed with the requirement that they give the correct bulk and interfacial equilibrium fluctuations. The resulting diffusion equation for a mixture in isothermal conditions is

$$
\frac{\partial C_{\nu}}{\partial t}=D_{\nu} \Delta C_{\nu}-\nabla \cdot \mathbf{q}^{\nu}(\mathbf{r}, t),
$$

with the following boundary conditions at the interface:

$$
\begin{gathered}
\left(C_{L}-C_{S}\right) v_{n}=\hat{\mathbf{n}} \cdot\left[D_{S} \boldsymbol{\nabla} C_{S}-D_{L} \boldsymbol{\nabla} C_{L}\right]+\hat{\mathbf{n}} \cdot\left[\mathbf{q}^{L}-\mathbf{q}^{S}\right], \\
m_{E} C_{L}+\Gamma \kappa+\frac{v_{n}}{\mu}=T_{M}-T+\eta(\mathbf{r}, t),
\end{gathered}
$$

where $\nu=S, L$ denotes the phase, $C_{\nu}$ is the concentration, $T_{M}-T$ is the undercooling, $D_{\nu}$ is the diffusion coefficient, $m_{E}$ is the absolute value of the (negative) $T\left(C_{L}\right)$ slope of the coexistence curve, and $\kappa, \hat{\mathbf{n}}$, and $v_{n}$ are the curvature, the normal unitary vector, and the normal velocity of the interface, respectively. $\Gamma=\sigma T / L, \sigma$ is the surface energy and $L$ 


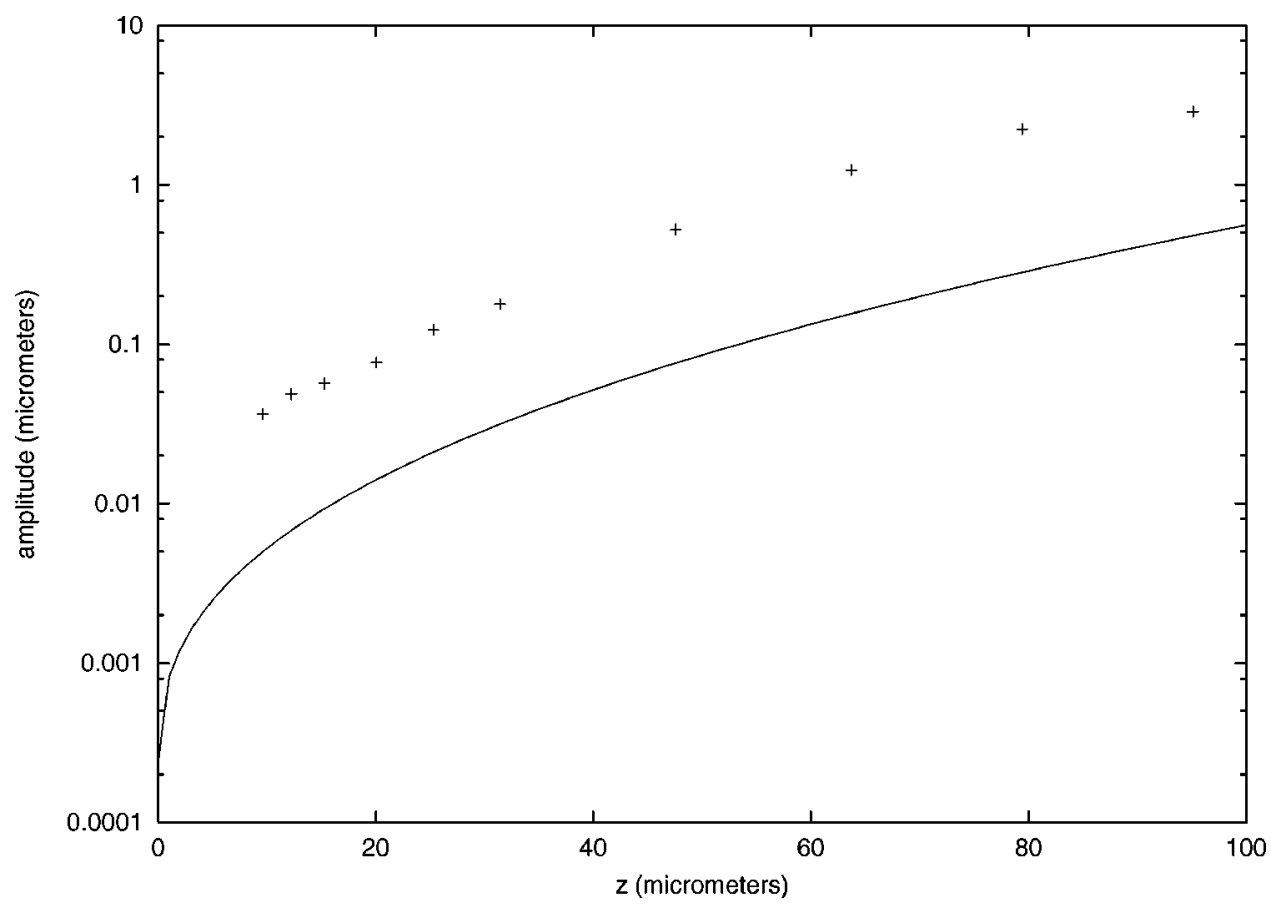

FIG. 1. Amplitude vs distance to the tip $z$ of the sidebranching induced by internal noise in the experiments of Ref. [18]. Line: theoretical prediction of Eq. (2.10) with an overestimated value of $\bar{S}$; crosses: experimental results (taken with permission from Ref. [18]). Quantities are expressed in micrometers. the latent heat per unit volume. $\mathbf{q}$ and $\eta$ are random forces whose statistical properties are given by

$$
\begin{aligned}
& \left\langle q_{i}^{\nu}(\mathbf{r}, t) q_{j}^{\nu}\left(\mathbf{r}^{\prime}, t^{\prime}\right)\right\rangle=2 D_{\nu} C_{\nu}(\mathbf{r}, t) \delta\left(\mathbf{r}, \mathbf{r}^{\prime}\right) \delta\left(t-t^{\prime}\right) \delta_{i j} \\
& \left\langle\eta_{i}^{\nu}\left(\mathbf{r}_{\perp}, t\right) \eta_{j}^{\nu}\left(\mathbf{r}_{\perp}^{\prime}, t^{\prime}\right)\right\rangle=2 \frac{k_{B} T^{2}}{\mu L} \frac{\delta\left(\mathbf{r}_{\perp}, \mathbf{r}_{\perp}^{\prime}\right) \delta\left(t-t^{\prime}\right)}{\sqrt{1+\left|\nabla_{\perp} \xi\left(\mathbf{r}_{\perp}, t\right)\right|}}
\end{aligned}
$$

where the interface is parametrized by the vector $\mathbf{r}_{\perp}+\xi \hat{\mathbf{n}}$.

These equations can be mapped under several approximations into the corresponding Langevin model for free solidification of a pure substance $[26,41,42]$. First we assume a constant concentration gap in the mass conservation Eq. (2.2), i.e., $C_{L}-C_{S} \equiv \Delta C \approx \Delta C^{\mathrm{eq}}$, the value corresponding to equilibrium at temperature $T$. This is in principle valid for small curvatures and velocities. A similar approximation is assumed in the intensity of the bulk noise $q_{i}^{\nu}(\mathbf{r}, t)$, substituting $C_{\nu}(\mathbf{r}, t)$ by the equilibrium value $C_{\nu}^{\mathrm{eq}}$ in Eq. (2.4). Furthermore in the intensity of the interfacial noise $\eta_{i}^{\nu}\left(\mathbf{r}_{\perp}, t\right)$ we employ the Clausius-Clapeyron equation for dilute alloys [42] to make the substitution $k_{B} T^{2} / \mu L \approx C_{L}^{\mathrm{eq}} m_{E} / \mu \Delta C^{\mathrm{eq}}$ in Eq. (2.5). Within these approximations the process of isothermal solidification of an alloy is equivalent (including thermodynamical fluctuations) to the (heat diffusion controlled) solidification of a pure substance, whose specific heat, latent heat, and melting temperature are given by

$$
\begin{gathered}
c_{p}=k_{B} \frac{\left(C_{L}^{\mathrm{eq}}\right)^{3}}{\left(\Delta C^{\mathrm{eq}}\right)^{2}}, \\
L=k_{B} \frac{\left(C_{L}^{\mathrm{eq}}\right)^{3}}{\Delta C^{\mathrm{eq}}} m_{E},
\end{gathered}
$$

$$
\bar{T}_{M}=\frac{\left(C_{L}^{\mathrm{eq}}\right)^{2}}{\Delta C^{\mathrm{eq}}} m_{E}
$$

where the diffusion field is now a temperature field given by

$$
\bar{T}(\mathbf{r}, t)-\bar{T}_{M}=\left(C_{\nu}-C_{\nu}^{\mathrm{eq}}\right) m_{E}
$$

and the rest of parameters remain unchanged. This can be checked by direct substitution in the Langevin equations. Therefore, the sidebranching induced by thermodynamical fluctuations should be the same in both situations. Sidebranching amplitude is predicted to depend on the distance $z$ along the dendrite axis as [17]

$$
A(z)=\rho \bar{S} \exp \left(\frac{2}{3}\left[\frac{x_{0}^{3}(z)}{3 \sigma^{*} z \rho^{2}}\right]^{1 / 2}\right)
$$

where $\rho$ is the tip radius, $x_{0}(z)$ is the shape of the dendrite, and the operating mode of the dendrite is given by the parameter $\sigma^{*}$ defined by

$$
\sigma^{*}=2 D d_{0} / \rho^{2} V
$$

where $V$ is the selected velocity and $d_{0}$ is the capillary length. The dimensionless noise amplitude, for a $d$-dimensional thermal dendrite, is then known to be $[17,26]$

$$
\bar{S}^{2}=\frac{2 k_{B} T_{M}^{2} c_{p} D}{L^{2} \rho^{1+d} V} \text {. }
$$




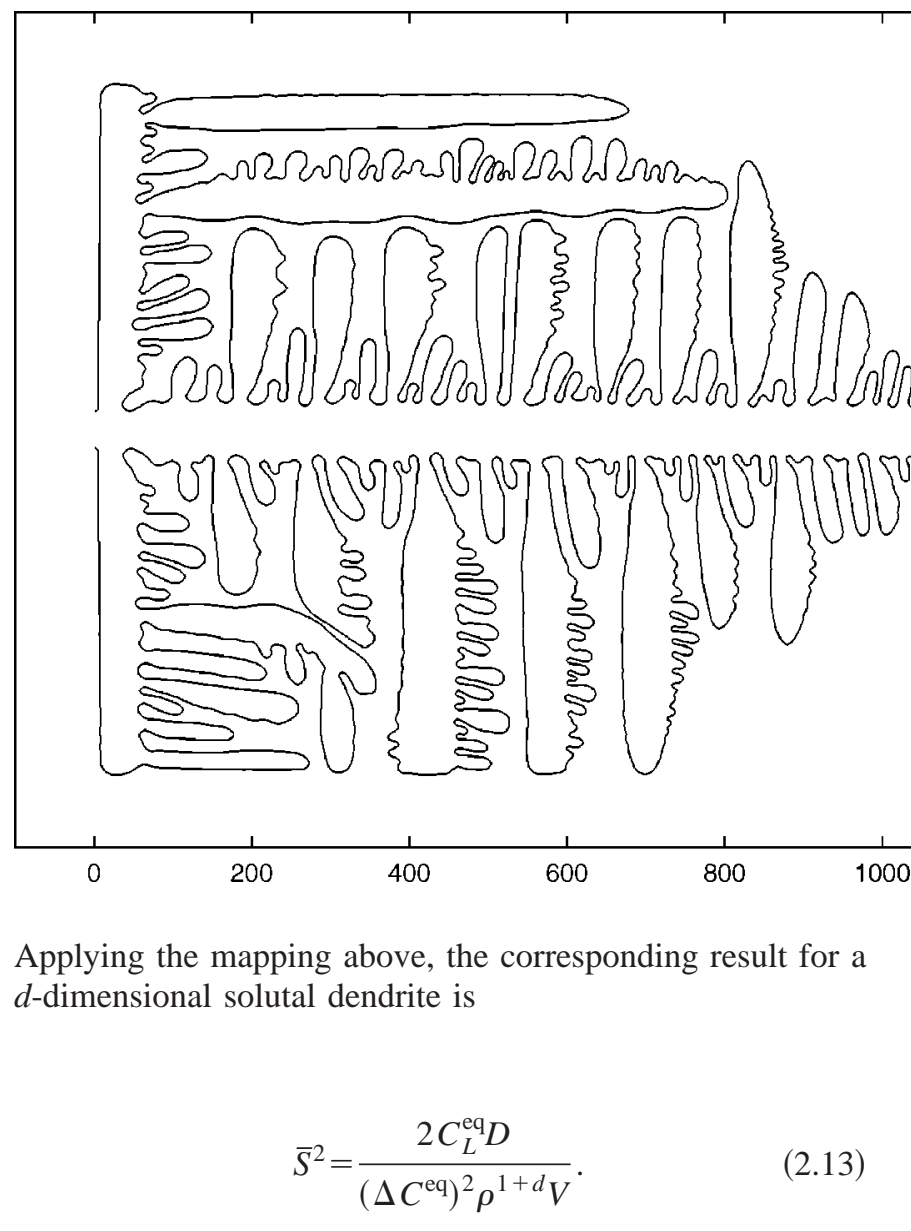

This result will be used below to compare the prediction for the case of internal noise with experiments on solutal dendrites, for which there are not many quantitative experimental results available. We focus on the experiments performed in Ref. [18] with ammonium bromide dendrites growing from supersaturated aqueous solution in isothermal conditions. In this experiment the precipitate front advances by incorporating solute particles instead of rejecting them, which makes it differ by several details from standard solutal solidification. Nevertheless, the above result [Eq. (2.13)] can be obtained by slightly adapting the performed mapping. In this case the system is on the high concentration side of the phase diagram of the mixture, for which $T_{M}$ is that of the solvent, no longer close to the temperature of the experiment. In this case it is convenient to write the Gibbs-Thomson equation as

$$
m_{E}\left(C_{\infty}-C_{L}\right)+\Gamma \kappa+\frac{v_{n}}{\mu}=T_{S}-T+\eta(\mathbf{r}, t),
$$

where $C_{\infty}$ is the concentration of the dilution, $T_{S}$ is the saturation temperature for that concentration, and $m_{E}$ is now the (positive) $T\left(C_{L}\right)$ slope. The same results of Eqs. (2.10) and (2.13) are obtained by applying the mapping of Eqs. (2.6), (2.7), and (2.8) with a diffusion field
FIG. 2. Dendrite obtained with the phase-field model with a noise term included, as is described in the text. Ticks denote number of grid points. where we have used the relation $T_{S}-T=m_{E}\left(C_{\infty}-C_{L}^{\mathrm{eq}}\right)$.

Now we compare the prediction of Eqs. (2.10) and (2.13) with the experimental results on supersaturated solutions of Ref. [18]. These experiments were performed at a supersaturation $\Delta=\left(C_{\infty}-C_{\mathrm{eq}}\right) /\left(C_{S}-C_{\mathrm{eq}}\right)=0.007$ and a saturation temperature of $56^{\circ} \mathrm{C}$. The characteristics of the selected dendrite are $\rho=4.0 \mu \mathrm{m}, V=1.44 \mu \mathrm{m} / \mathrm{s}$, and $\sigma^{*}=0.081$. We have employed a value of $D=2.6 \times 10^{-5} \mathrm{~cm}^{2} / \mathrm{s}$ and the values corresponding to a temperature of $100^{\circ} \mathrm{C}$ for the equilibrium concentrations $C_{L}^{\mathrm{eq}}=0.99 \times 10^{4} N_{A}$ molecules $/ \mathrm{m}^{3}$ and $C_{S}^{\mathrm{eq}}=2.48 \times 10^{4} N_{A}$ molecules $/ \mathrm{m}^{3}$. Since this temperature is much higher than that of the experiment, the resulting value $\bar{S}=5.96 \times 10^{-5}$ constitutes an overestimation of the theoretical value.

In Fig. 1 we show the calculated amplitude $A(z)$ of the sidebranching induced by internal fluctuations for this value of $\bar{S}$. We consider the theoretically predicted shape $x(z)$ $=\left(\frac{5}{3} z\right)^{3 / 5}$, as was considered in Ref. [17]. The result corresponding to the actual temperature of the experiment would be placed below the represented curve. In the same figure we plot the experimental results of Ref. [18]. We see that experimental amplitudes are approximately one order of magnitude larger than the overestimated theoretical values.

Therefore the predicted amplitude of the sidebranching when it is due to statistical noise is, for the experiments of Ref. [18], at least one order of magnitude smaller than the amplitude experimentally observed. Thus, we are led to conclude that thermodynamical fluctuations are not enough to explain the sidebranching amplitude in some experiments. In this estimation we have assumed three-dimensional dendrites even though the experiments of Ref. [18] are intended to be quasi-two-dimensional. The analogous calculations in two 
dimensions show an even lower sidebranching amplitude, i.e., a larger discrepancy with the experiments.

There is a shortcoming in the predictions above when applied to supersaturated experiments. By their own nature, supersaturated dilutions are not in the diluted limit, as assumed in the theoretical analysis [42]. In the experiments of Ref. [18] the concentration is as high as $16 \%$ of solute molecules. Indeed, the whole Langevin formalism is constructed in order to guarantee that the concentration fluctuations in a small volume $\Delta V$ is

$$
\left\langle\left(\Delta C_{\nu}\right)^{2}\right\rangle=\frac{C_{\nu}}{\Delta V},
$$

which is the equilibrium value for diluted solutions. In fact, for a concentrated solution Eq. (2.16) should be replaced by

$$
\left\langle\left(\Delta C_{\nu}\right)^{2}\right\rangle=\frac{1}{\Delta V} \frac{T}{\left(\frac{\partial \mu}{\partial C}\right)_{P, T}} .
$$

Since the sidebranching amplitude in supersaturated solutions has been found to be at least 1 order of magnitude larger than predicted in the diluted approximation, one concludes that it would be necessary that the derivative of the chemical potential is 2 orders of magnitude greater than that given by the diluted approximation $\partial \mu / \partial C \approx T / C$ in order to explain the experiments by internal noise. Therefore, most likely this internal noise is really not strong enough to account for the observed sidebranching. As we are not aware of quantitative data on thermodynamical properties of supersaturated solutions that would permit us to improve the estimations above, a definitive answer on the amplitude of sidebranching in these dendrites remains open. In any case these results call for experimental quantitative measurements in solutal dendrites grown in diluted conditions, where Eq. (2.13) properly applies.

\section{MODEL AND NUMERICAL PROCEDURE}

We have performed simulations of dendritic growth by employing a phase-field model for solidification. In this model both phases and their interface are treated indistinctly, and discriminated by an effective nonconserved order parameter or phase field $\phi$, which takes different values in each phase ( 0 and 1 in our simulations). This field changes smoothly across an interface region of finite thickness, and its dynamics is coupled to that of the diffusion field in such a way that the sharp-interface model is recovered in the limit of vanishing interface thickness, controlled by a new small parameter $\epsilon$. The equations of the model read explicitly

$$
\begin{aligned}
\epsilon^{2} \tau(\theta) \frac{\partial \phi}{\partial t}= & \phi(1-\phi)\left(\phi-\frac{1}{2}+30 \epsilon \beta \Delta u \phi(1-\phi)\right) \\
& -\epsilon^{2} \frac{\partial}{\partial x}\left[\eta(\theta) \eta^{\prime}(\theta) \frac{\partial \phi}{\partial y}\right] \\
& +\epsilon^{2} \frac{\partial}{\partial y}\left[\eta(\theta) \eta^{\prime}(\theta) \frac{\partial \phi}{\partial x}\right]+\epsilon^{2} \nabla\left[\eta^{2}(\theta) \nabla \phi\right],
\end{aligned}
$$

$$
\frac{\partial u}{\partial t}+\frac{1}{\Delta}\left(30 \phi^{2}-60 \phi^{3}+30 \phi^{4}\right) \frac{\partial \phi}{\partial t}=\nabla^{2} u+\psi(x, y, t),
$$

where $u(\mathbf{r}, t)$ is the diffusion field and $\Delta$ is the dimensionless undercooling. Lengths are scaled in some arbitrary reference length $\omega$, while times are scaled by $\omega^{2} / D$. In these equations $\theta$ is the angle between the $x$ axis and the gradient of the phase field. $\eta(\theta)$ is the anisotropy of the surface tension. The anisotropy of the kinetic term is then given by $\tau(\theta) / \eta(\theta) . \beta$ is equal to $\sqrt{2} \omega / 12 d_{0}$ and $d_{0}$ is the capillary length.

The external noise is introduced through the additive term $\psi$ in the heat equation. This choice is not unique and is justified here only for simplicity. Because of its external origin, the noise is not assumed to satisfy a fluctuationdissipation relation. Furthermore, it is generally assumed to be nonconserved, as opposed to the more usual case of thermal noise, which would enter the model equations as a stochastic current (i.e., conserved noise) in the heat equation, and an additional stochastic term in the phase-field equation [26]. In our simulations the noise term is evaluated at each uncorrelated cell of lateral size $\Delta x$ simply as $I \cdot r$, where $I$ denotes the amplitude of the noise, and $r$ is a uniform random number in the interval $[-0.5,0.5]$. The phase-field model equations have been solved on rectangular lattices using first-order finite differences on a uniform grid with mesh spacing $\Delta x$. An explicit time-differencing scheme has been used to solve the equation for $\phi$, whereas for the $u$ equation the alternating-direction-implicit (ADI) method was chosen, which is unconditionally stable [29]. The kinetic term has been taken as isotropic, which leads to $\tau(\theta)=m \eta(\theta)$ with constant $m$. A fourfold free energy anisotropy $\sigma=\sigma(0)[1$ $+\gamma \cos (4 \theta)]$ has been considered. This gives rise to dendrites growing with perpendicular side branches. The values of $\gamma$ taken were always smaller than 0.0625 , which ensured that we obtained rounded shapes such as a parabola because forbidden directions were avoided [5].

The growth morphologies were obtained by setting a small vertical seed $(\phi=0, u=0)$ in the center of either of the two shortest sides of the system and imposing $\phi=1$ and $u=-1$ on the rest of the system. We have considered symmetric boundary conditions for $\phi$ and $u$ on the four sides of the system, although they do not influence the results presented in this paper.

We have used a set of phase-field model parameters that give rise to a growing needle without sidebranching for every anisotropy $\gamma$ considered when no noise is added to the simulations. This ensures that the sidebranching observed when noise is present is not due to computational rounding. The fixed phase-field model parameters for all the simulations are $\beta=400, m=20$, and $\epsilon=0.003$. The values of $\Delta$ and $\gamma$ have been varied in the range $0.5-0.7$ and 0.045 -0.060 , respectively. The noise amplitude is varied in the range $5-150$. The time and spatial discretizations were kept constant in all the simulations with values $\Delta x=0.01$ and $\Delta t=10^{-4}$. Two system sizes were used in the simulations. A 

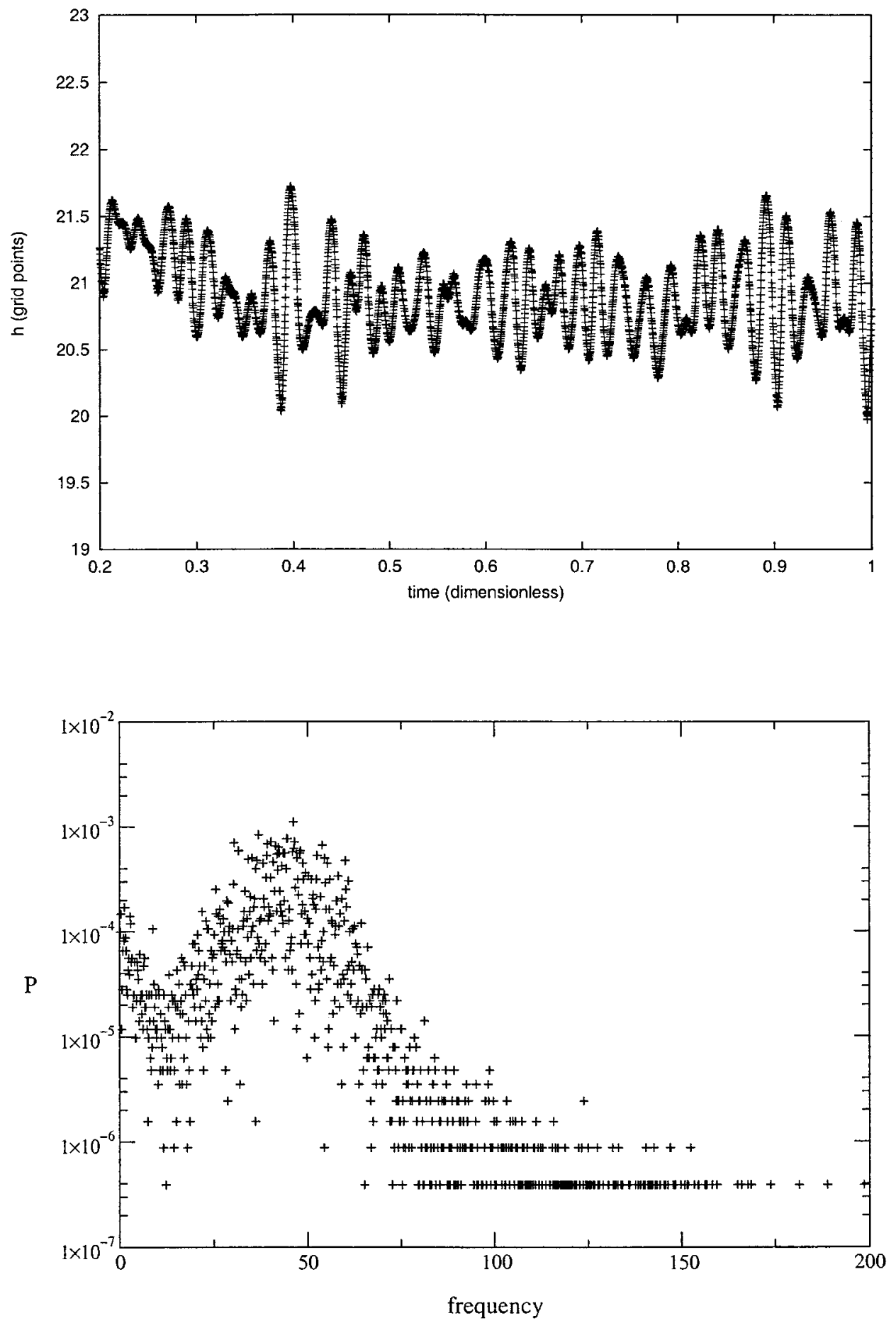

FIG. 3. Half-width $h_{z}(t)$ of the dendrite and its power spectrum $P_{z}(f) \quad(P$ in square grid points times dimensionless time and frequency in cycles per dimensionless time) for $z=40$ grid points. system with $1000 \times 1500$ grid points was used to observe fully developed sidebranching including tertiary arms and to have enough statistics in order to compare with measurements of the sidebranching correlation at both sides of the dendrite. Additionally a $500 \times 500$ grid points system was used when the data sets did not require very extensive statistics. In Fig. 2 a growing dendrite is shown with $\gamma$ $=0.045, \Delta=0.6, I=11$ at a time $t=1.5$. The velocity and the radius of the tip are very weakly affected when noise is introduced. However, side branches appear at both sides of the main dendrite, yielding approximately a $90^{\circ}$ angle, as was observed in Ref. [18]. Further down the tip one can clearly observe competition between branches which gives rise to a coarsening effect. When branches reach the vertical boundaries of the system, they are stopped by the effect of the symmetric boundary conditions. This did not affect the measurements presented in this paper, where we have focused on the region between the tip and a point approximately 150 grid points down from the tip (grid points are marked on the axes of Fig. 2). This region corresponds approximately to that considered in the experimental work in Ref. [18] and, moreover, it is an appropriate region in order 

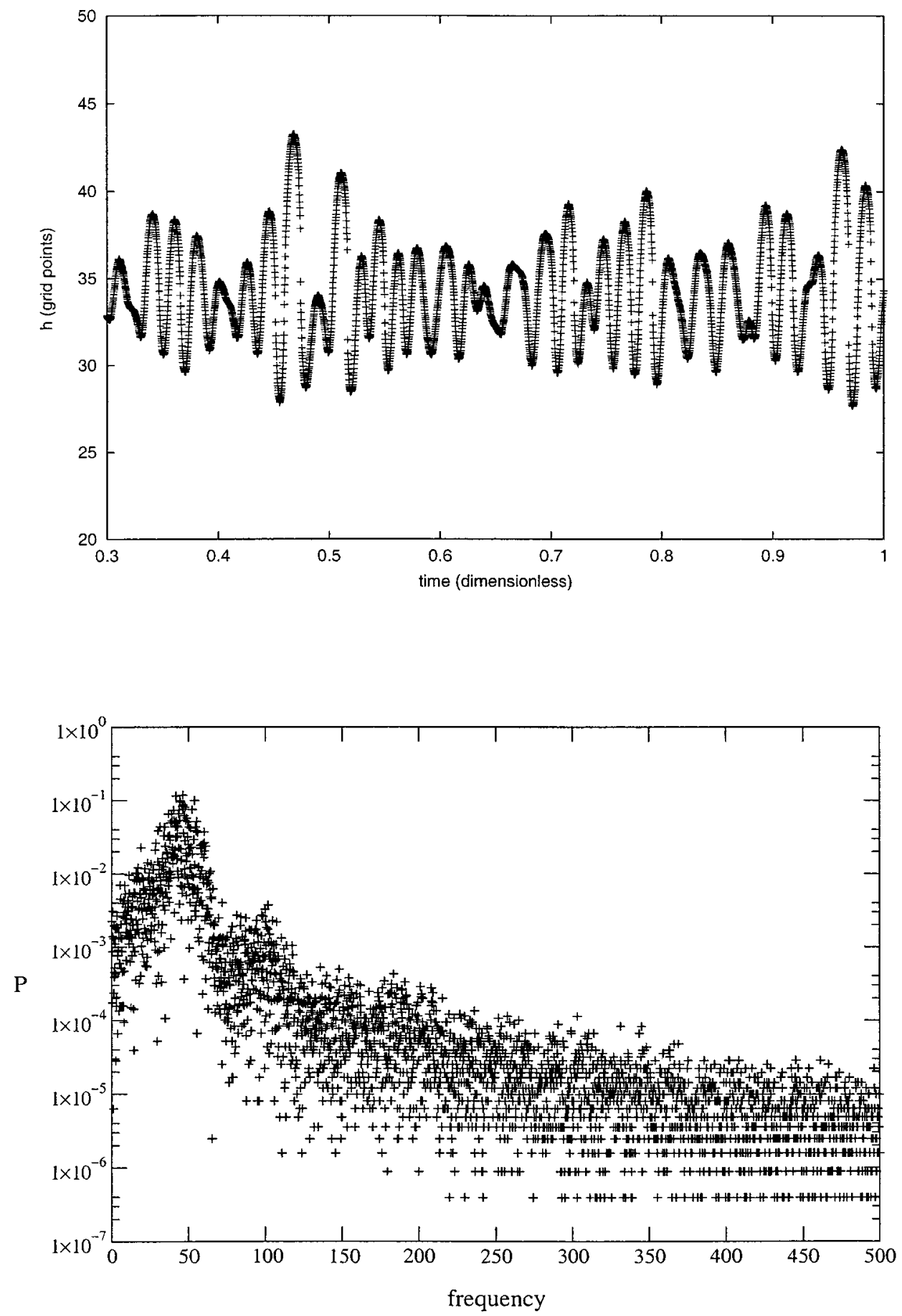

FIG. 4. Half-width $h_{z}(t)$ of the dendrite and its power spectrum $P_{z}(f)$ ( $P$ in square grid points times dimensionless time and frequency in cycles per dimensionless time) for $z=100$ grid points. to compare the behavior of sidebranching with the analytical results obtained from linear perturbation theory. In the longer runs, and in order to avoid working with unnecessarily large systems, we have performed periodic shifts of the complete system. We have checked that this did not affect the results of the simulation.

\section{SIDEBRANCHING CHARACTERISTICS}

In order to study the sidebranching induced by noise we have measured the half-width $h_{z}(t)$ of the dendrite at various distances $z$ behind the tip as a function of time. In order to have a comparable amount of data as in Ref. [18] (they recorded around 240 oscillations of the amplitude for each $z$ ) we needed to simulate a dendrite four times longer $(t=6)$ than the one shown in Fig. 2. The half width of the dendrite as a function of time at a distance $z=40$ grid points from the tip as well as its power spectrum $P_{z}(f)$ are shown in Fig. 3. The same type of data corresponding to a point further from the tip ( $z=100$ grid points) is shown in Fig. 4. The data used to compute the power spectra were six times the lengths shown. Both sets of data have a strong resemblance to those obtained experimentally [18]. We also computed the crosscorrelation function 


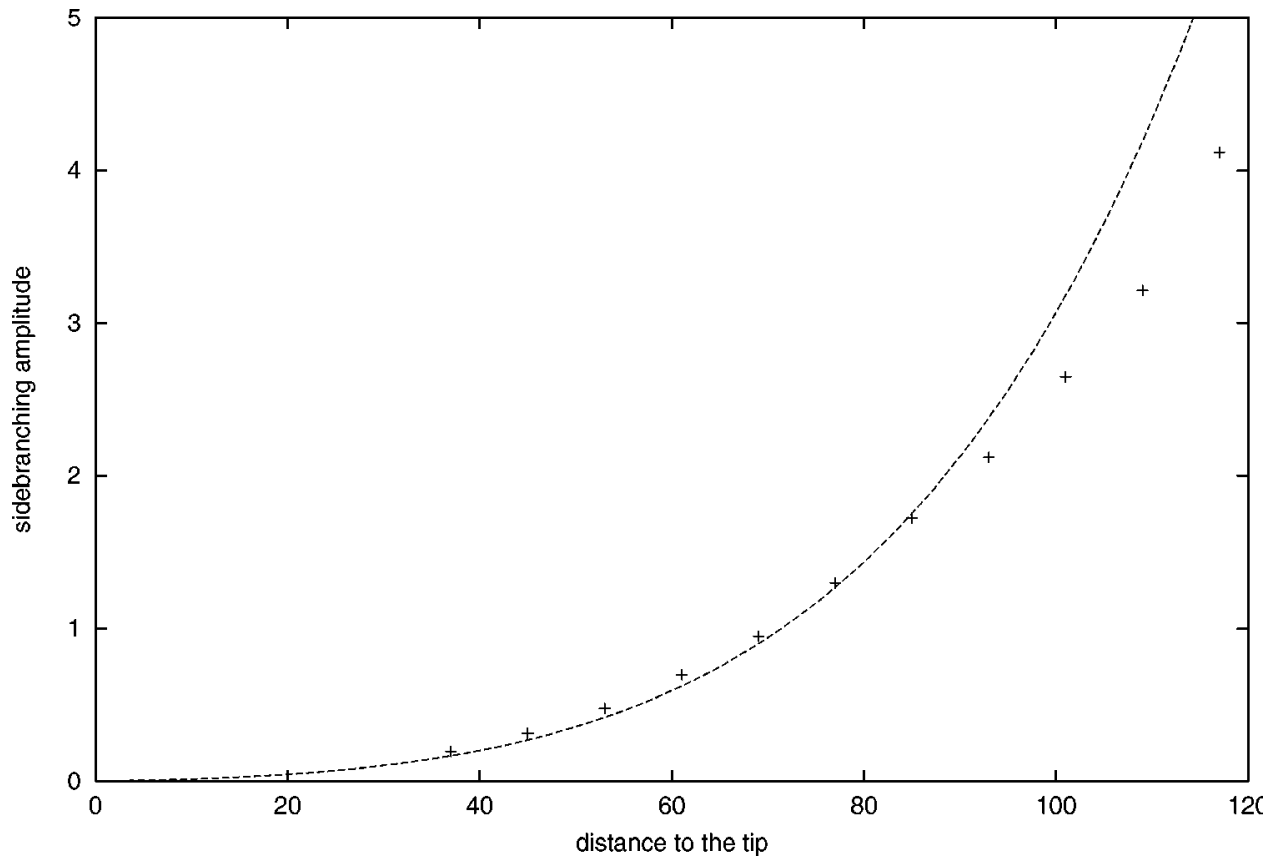

FIG. 5. Square root of the area under the spectral peak as a function of $z$ (both in grid points). The line indicates exponential growth with $z^{2 / 5}$.

$$
C\left(t^{\prime}\right)=\left\langle\left[h_{L z}\left(t+t^{\prime}\right)-\bar{h}_{L z}\right]\left[h_{R z}(t)-\bar{h}_{R z}\right]\right\rangle / \sigma_{L} \sigma_{R},
$$

where $h_{L z}(t), h_{R z}(t), \sigma_{L}$, and $\sigma_{R}$ are the half-width functions and their standard deviations for the two sides of the dendrite at the same distance from the tip. We found that $C(0)$ is approximately 0.4 for points very close to the tip and that its value decreases very quickly to 0 when increasing $z$. Moreover, in the points closer to the tip, $C\left(t^{\prime}\right)$ drops to zero after six oscillations, that is, the time to nucleate six side branches. The same behavior was observed in the experiments in Ref. [18]. In simulations where we used a smaller data set, the values of $C\left(t^{\prime}\right)$ were larger than those found in the experiments, as was to be expected.

In addition to the predictions for the amplitude commented on above, the behavior of the wavelength is another main feature of sidebranching. It depends very weakly on $z$ $[17,26]: \lambda \sim z^{1 / 5}$. Despite the fact that experiments $[18,19]$ showed the predicted dependence of the amplitude on $z$, the same frequency of the spectral peak for different $z$ was found.

In order to observe the dependence of the sidebranching amplitude on $z$ in the simulations, the phase-field model simulations were run on a $500 \times 500$ grid points system. In Fig. 5 we show the square root of the area under the spectral peak as a function of $z$. This representation gives the amplitude behavior of the sidebranching as a function of the distance to the tip. The data were obtained with $\gamma=0.045, \Delta$ $=0.6, I=19$ until a time $t=0.5$. The amplitude is found to increase exponentially with $z^{2 / 5}$ for distances $z<100$. Thus the behavior of the data obtained in the simulations is consistent with the linear analysis $[17,26]$ up to a certain value of $z$. In Ref. [18] similar behavior was found in the linear regime, although they found a saturation of the amplitude further down from the tip. This could be due to some boundary effects which stop the growth of the side branches.
The dependence of the sidebranching wavelength on the distance to the tip was studied with the data recorded in the same simulation as for Fig. 5 and after performing the power spectrum. The wavelength was found to remain constant in the interval of considered $z$, which is consistent with the observations in Ref. [26].

\section{CONCLUDING REMARKS}

We have studied the sidebranching induced by fluctuations in dendritic growth, for which there is good quantitative agreement between theory and experiment for thermal dendrites (i.e., controlled by heat diffusion). This agreement has only been qualitative for solutal dendrites, as there was no prediction available for their sidebranching amplitudes. In order to be able to perform a quantitative comparison, we have obtained an estimation of sidebranching amplitudes originated by internal fluctuations in solutal diluted dendrites. The resulting values appear as much smaller (at least one order of magnitude) than those observed in some experiments (performed in concentrated solutions). This can be attributed to the effect of other noise sources, of an external origin. To confirm this conclusion, it would be necessary to make use of quantitative experimental results obtained in more diluted conditions.

We have obtained the effects of noninternal fluctuations on dendritic sidebranching by introducing nonconserved noise in a phase-field model for solidification. Our simulations qualitatively reproduce well previous experimental and analytical results. In particular, we have reproduced the dependence of the sidebranching amplitude on the distance from the tip, confirming the validity of what was previously obtained with a linear theory including internal noise. Thus, the qualitative behavior of the sidebranching amplitude, 
when this is due to the selective amplification of fluctuations, is basically independent of the origin of the noise. In conclusion, qualitative concordance between experimental results does not directly imply a common source of fluctuations and therefore, a careful quantitative study of sidebranching activity may help to elucidate the origin of the dominant noise in each experiment.

The phase-field model appears to be a versatile method to study dendritic growth in general and sidebranching characteristics in particular. It has been shown to be adapted to take into account thermodynamical fluctuations [25-27], but alternative ways to introduce noise (such as the one employed in this work) appear to be appropriate to qualitatively reproduce the behavior of sidebranching when the noise is of an external origin. Our results on sidebranching can also be helpful for further simulations where qualitatively realistic sidebranching needs to be distinguished from that generated by numerical noise due to the round-off of the computer.

\section{ACKNOWLEDGMENTS}

The authors wish to thank Professor W. Van Saarloos and Dr. T. Toth Katona for many fruitful discussions. R.G.C. is indebted to the Lorentz Institut for its kind hospitality and assistance. The authors thank the Dirección General de Investigación Científica y Técnica (Spain) (Projects No. BFM2000-0624-C03-02, BFM2000-0628-C03-01, and BXX2000-0638-C02-02), Comissionat per a Universitats i Recerca (Spain) (Projects No. 1999SGR00145 and 1999SGR00041), and European Commission (TMR Network Project No. ERBFMRXCT96-0085) for financial support. We also acknowledge the Center de Supercomputació de Catalunya (CESCA) for computing support.
[1] J. S. Langer, in Chance and Matter, 1986 Les Houches Lectures, edited by J. Souletie, J. Vannimenus, and R. Storra (North Holland, Amsterdam, 1987), pp. 629-711.

[2] Dynamics of Curved Fronts, edited by P. Pelcé (Academic Press, New York, 1988).

[3] E. Ben-Jacob and P. Garik, Nature (London) 343, 523 (1990).

[4] E. A. Brener and V. I. Melnikov, Adv. Phys. 40, 53 (1991).

[5] Solids Far from Equilibrium, edited by C. Godrèche (Cambridge University Press, Cambridge, 1992).

[6] Handbook of Crystal Growth, edited by D. T. J. Hurle (NorthHolland, Amsterdam, 1993), Vol. 1B.

[7] Spatio Temporal Patterns, Santa Fe Institute Studies in the Science of Complexity Vol. XXI, edited by P. E. Cladis and P. Palffy-Muhoray (Addison Wesley, New York, 1994).

[8] J. S. Langer and H. Müller-Krumbhaar, Phys. Rev. A 27, 499 (1983).

[9] O. Martin and N. Goldenfeld, Phys. Rev. A 35, 1382 (1987).

[10] J. C. LaCombe, M. B. Koss, C. Giummarra, J. E. Frei, A. O. Lupulescu, and M. E. Glicksman, in Proceedings of the Sitges Conference Coherent Structures in Classical Systems (Springer Verlag, Berlin, in press).

[11] W. van Saarloos, B. Caroli, and C. Caroli, J. Phys. I 3, 741 (1993).

[12] R. Pieters and J. S. Langer, Phys. Rev. Lett. 56, 1948 (1986).

[13] D. A. Kessler and H. Levine, Europhys. Lett. 4, 215 (1987).

[14] M. N. Barber, A. Barbieri, and J. S. Langer, Phys. Rev. A 36, 3340 (1987).

[15] R. Pieters, Phys. Rev. A 37, 3126 (1988).

[16] J. S. Langer, Phys. Rev. A 36, 3350 (1987).

[17] E. Brener and D. Temkin, Phys. Rev. E 51, 351 (1995).

[18] A. Dougherty, P. D. Kaplan, and J. P. Gollub, Phys. Rev. Lett. 58, 1652 (1987).

[19] X. W. Qian and H. Z. Cummins, Phys. Rev. Lett. 64, 3038 (1990).

[20] Ph. Bouissou, A. Chiffaudel, B. Perrin, and P. Tabeling, Europhys. Lett. 13, 89 (1990).

[21] U. Bisang and J. H. Bilgram, Phys. Rev. Lett. 75, 3898 (1995).
[22] U. Bisang and J. H. Bilgram, Phys. Rev. E 54, 5309 (1996).

[23] E. Hürlimann, R. Trittibach, U. Bisang, and J. H. Bilgram, Phys. Rev. A 46, 6579 (1992).

[24] L. M. Williams, M. Muschol, X. Qian, W. Losert, and H. Z. Cummins, Phys. Rev. E 48, 489 (1993).

[25] S. G. Pavlik and R. F. Sekerka, Physica A 268, 283 (1999).

[26] A. Karma and W.-J. Rappel, Phys. Rev. E 60, 3614 (1999).

[27] S. G. Pavlik and R. F. Sekerka, Physica A 277, 415 (2000).

[28] R. Kobayashi, Physica D 63, 410 (1993).

[29] A. A. Wheeler, B. T. Murray, and R. J. Schaefer, Physica D 66, 243 (1993).

[30] G. B. McFadden, A. A. Wheeler, R. J. Braun, S. R. Coriell, and R. F. Sekerka, Phys. Rev. E 48, 2016 (1993).

[31] S.-L. Wang, R. F. Sekerka, A. A. Wheeler, B. T. Murray, S. R. Coriell, R. J. Braun, and G. B. McFadden, Physica D 69, 189 (1993).

[32] R. J. Braun, G. B. McFadden, and S. R. Coriell, Phys. Rev. E 49, 4336 (1994).

[33] B. T. Murray, A. A. Wheeler, and M. E. Glicksman, J. Cryst. Growth 154, 386 (1995).

[34] S.-L. Wang and R. F. Sekerka, Phys. Rev. E 53, 3760 (1996).

[35] R. González-Cinca, L. Ramírez-Piscina, J. Casademunt, A. Hernández-Machado, L. Kramer, T. Tóth Katona, T. Börzsönyi, and Á. Buka, Physica D 99, 359 (1996).

[36] T. Tóth-Katona, T. Börzsönyi, Z. Váradi, J. Szabou, Á. Buka, R. González-Cinca, L. Ramírez-Piscina, J. Casademunt, and A. Hernández-Machado, Phys. Rev. E 54, 1574 (1996).

[37] A. Karma and W. J. Rappel, Phys. Rev. E 53, 3017 (1996).

[38] R. González-Cinca, L. Ramírez-Piscina, J. Casademunt, A. Hernández-Machado, T. Tóth Katona, T. Börzsönyi, and Á. Buka, J. Cryst. Growth 193, 712 (1998).

[39] T. Börzsönyi, Á. Buka, and L. Kramer, Phys. Rev. E 58, 6236 (1998).

[40] T. Börzsönyi, T. Tóth-Katona, Á. Buka, and L. Gránśy, Phys. Rev. Lett. 83, 2853 (1999).

[41] A. Karma, Phys. Rev. Lett. 70, 3439 (1993).

[42] A. Karma, Phys. Rev. E 48, 3441 (1993). 